\title{
Medication Management with Panic Disorder in the Exercise State: Some Prescribing Guidelines
}

\section{To the Editor:}

January 2, 2007

The question of whether exercise has an acute antipanic activity versus inducing panic symptoms is not without controversy. ${ }^{1,2} \mathrm{~A}$ literature search revealed no data regarding the utility of serotonin reuptake inhbitors regarding possible modulating effects during exercise. Here is a case presentation that discusses the benefits of medication in panic disorder regarding the ability to tolerate higher intensities of exercise.

Mr. Z was a 33-year-old African American male who endorsed panic symptoms, including dizziness, palpitations, sense of fear, shortness of breath, shaking, and tachycardia, which would occur "out of the blue" and were not situation specific. When exercising, he noticed that an increased heart rate was a trigger for further and increased panic symptoms. A medical evaluation including a complete cardiac assessment was within normal. Sertraline was initiated and tapered up to $200 \mathrm{mg} /$ day, which resulted in full remission of his panic symptoms while in a nonexercise state. Alprazolam was initiated at 0.5 $\mathrm{mg} /$ day for breakthrough episodes early in treatment and was discontinued once his symptoms were in remission. However, prior to sertraline, a heart rate $>130$ beats per minute was followed by increased dizziness which resulted in a full blown panic attack. Post-sertraline, panic symptoms did not occur until Mr. Z's heart rate $>150$ beats per minute. This difference was verified by pre- and post-treadmill tests using the Bruce protocol. This extension of the panic-free symptoms allowed Mr. $Z$ to exercise at a moderate intensity level and pass his sub-maximal (AstrandRhyming protocol) ergometry fitness test.
This case suggests that medication treatment may increase the threshold for panic symptoms during exercise. Also, full remission of symptoms while in a non-exercise state may not necessarily translate to an exercise setting. Furthermore, heart rate monitoring during exercise, by noting the onset of panic symptoms may be a useful gauge of medication efficacy. This may be especially helpful when dealing withactivities that are more arduous. More research is necessary to fully substantiate these observations.

Sincerely,

Ashley B. Benjamin, MD, MA

\section{REFERENCES}

1. Strohle A, Feller C, Onken M, Godemann F, Heinz A, Dimeo F. The acute antipanic activity of aerobic exercise. Am J Psychiatry. 2005;162:2376-2378.

2. Cameron 0 . Hudson $C$. Influence of exercise on anxiety level in patients with anxiety disorders. Psychosomatics. 1986,27:720 -723.

Dr. Benjamin is medical director of the day-treatment and Operation Enduring Freedom/Operartion Iraqi Freedom programs at the Oklahoma City Veterans Affairs Medical Center and a clinical assistant professor in the Department of Psychiatry and Behavioral Sciences at the University of Oklahoma Health Sciences Center, Oklahoma City.

Acknowledgements: Thanks to Radwane Kesserwane, MD, internist at the OKC VA Medical Center, for his thoughtful suggestions.

Disclaimer: The opinions contained above are those of the author and do not reflect the opinions of the Department of Defense or the Department of Veterans Affairs.

Disclosure: Dr. Benjamin does not have an affiliation with or financial interest in any organization that might pose a conflict of interest.

Please send letters to the editor to: CNS Spectrums, c/o Eric Hollander, MD, 333 Hudson St., 7th Floor, New York, NY 10013; E-mail: vj@mblcommunications.com. 